\title{
Utility of cardiac magnetic resonance imaging in troponin-positive chest pain with non-obstructive coronary arteries: literature review
}

\author{
Jonan CY Lee *, Jeanie B Chiang, PP Ng, Boris CK Chow, YW Cheng, CY Wong
}

\section{A B S T R A C T}

There is no general consensus on the investigation and subsequent management of patients presenting with acute chest pain and elevated cardiac troponin levels, but with non-obstructive coronary arteries on angiography. Recent technological advances in cardiac magnetic resonance imaging have aided in the understanding of the underlying pathophysiology, allowing accurate diagnosis, prognostic information, and guidance for management in these patients. This article reviews the evidence supporting the usefulness of cardiac magnetic resonance imaging in patients with acute chest pain and elevated cardiac troponin levels, but with non-obstructive coronary arteries, and offers insights into the role and future development of this imaging modality in this disease.

\begin{tabular}{l} 
Hong Kong Med J 2021;27:266-75 \\
\hline https://doi.org/10.12809/hkmj208690 \\
1 JCY Lee *, FRCR, FHKAM (Radiology) \\
${ }^{1}$ JB Chiang, FRCR, FHKAM (Radiology) \\
${ }^{1}$ PP Ng, MB, ChB \\
${ }^{1}$ BCK Chow, MB, BS, FRCR \\
${ }^{2}$ YW Cheng, MRCP (UK), FHKAM (Medicine) \\
${ }^{2}$ CY Wong, MRCP (UK), FHKAM (Medicine) \\
${ }^{1}$ Department of Radiology and Imaging, Queen Elizabeth Hospital, Hong \\
Kong \\
${ }^{2}$ Department of Medicine, Queen Elizabeth Hospital, Hong Kong \\
* Corresponding author: jonanleecy@yahoo.com
\end{tabular}

\section{Introduction}

Patients presenting with acute coronary syndrome require immediate management with coronary angiography to identify the culprit coronary stenosis. ${ }^{1,2}$ A small subset of patients with suspected acute coronary syndrome may have angiographically nonobstructive coronary arteries, termed myocardial infarction with non-obstructive coronary arteries (MINOCA). ${ }^{3}$ Myocardial infarction with nonobstructive coronary arteries is indistinguishable in its clinical presentation from myocardial infarction with coronary artery disease. The normal coronary angiography results pose a dilemma to the managing physician because the underlying aetiology is not immediately apparent. Arriving at a diagnosis is challenging, with significant implications regarding patients' prognosis, management, and subsequent follow-up.

Myocardial infarction with non-obstructive coronary arteries is a distinct clinical entity with a prevalence of $6 \%(95 \%$ confidence interval $[\mathrm{CI}], 5 \%-7 \%)^{3}$ that deserves further meticulous investigation. Despite having non-obstructive coronary arteries, patients with MINOCA have an increased risk of experiencing major cardiovascular events (MACE) including death. Pasupathy et $\mathrm{al}^{3}$ reported $4.7 \%$ annual mortality in this group of patients, which is lower than for myocardial infarction with coronary artery disease (6.7\%) but much higher than in patients with stable chest pain ( $0.2 \%$ annual mortality).
Causes of MINOCA include acute myocardial infarction (AMI) with spontaneous recanalisation, coronary vasospasm, acute myocarditis, takotsubo cardiomyopathy, and other cardiomyopathies. ${ }^{4}$ Distinguishing between ischaemic and nonischaemic aetiologies is crucial in patients presenting with MINOCA, in order to tailor treatments accordingly, such as dual antiplatelet therapy and other secondary preventive medications for myocardial infarction, ${ }^{4}$ or heart failure medications for myocarditis or cardiomyopathies.

Cardiac magnetic resonance (CMR) imaging has been increasingly recognised as a first-line imaging modality in the management of patient presenting with MINOCA, to detect the aetiology in a timely manner. High-resolution cardiac images are acquired with tissue characterisation using different MR sequences.

The referral rate of CMR imaging for MINOCA has been low, with only $3 \%$ of all eligible patients undergoing further testing by CMR imaging in a retrospective study between 2000 and $2016 .{ }^{5}$ This is expected to change with the widespread availability and improved image quality of CMR imaging.

This review aims to summarise the current evidence regarding the use of CMR imaging in patients presenting with MINOCA, to demonstrate its use in various clinical scenarios, and to identify areas for future research. In particular, we review the optimal timing of CMR imaging. We also examine how CMR imaging may change or confirm the 
aetiology, offers prognostic information, and change management strategy.

We reviewed the medical literature in the PubMed database and Google Scholar, using the key terms 'MINOCA', 'myocardial infarction with non-obstructive coronary arteries', 'troponinpositive acute chest pain', 'non-obstructive coronary arteries', 'cardiac magnetic resonance', 'myocarditis', 'acute myocardial infarction' and 'takotsubo cardiomyopathy', for studies published up to April 2020. There was no language restriction. Abstracts were reviewed to determine their relevance to the aim of our review. Case reports and papers with unclear or inappropriate statistical methods were excluded. The discussion is based on, but not limited to, the search terms.

\section{Definition}

According to the European Society of Cardiology working group positional paper ${ }^{6}$ and the scientific statement from American Heart Association, ${ }^{7}$ MINOCA is adistinctclinical syndromecharacterised by evidence of $\mathrm{AMI},{ }^{8}$ but with no evidence of obstructive coronary artery disease on angiography (stenosis $<50 \%$ diameter in a major epicardial vessel). The term MINOCA refers to ischaemicrelated coronary disorders, namely plaque rupture, coronary vasospasm, microvascular dysfunction, distal embolisation, and coronary artery dissection. The American Heart Association states that it is imperative to exclude (a) clinically overt causes for elevated troponin (eg, sepsis, pulmonary embolism), (b) clinically over-looked obstructive disease, and (c) non-ischaemic disease that can mimic myocardial infarction (eg, myocarditis). In clinical practice, however, exclusion of non-ischaemic mechanism is often not straightforward. Elevated cardiac troponin levels signify myocardial injury, but the marker is non-specific for the underlying pathophysiological mechanism. For instance, acute myocarditis and takotsubo cardiomyopathy may present as MINOCA and may sometimes be even more frequent than ischaemic causes. ${ }^{9}$ Other non-cardiac causes such as pulmonary embolism or tumour infiltration may also present as MINOCA. ${ }^{6}$

Recently, the term troponin-positive chest pain with non-obstructive coronary arteries (TpNOCA) has been proposed to encompass all patients with ischaemic causes as well as non-ischaemic myocardial disorders and non-cardiac diseases. ${ }^{10}$ The Dutch ACS working group suggested that the term MINOCA can be understood as either myocardial infarction or myocardial injury with non-obstructive coronary arteries. ${ }^{11}$ Given the numerous underlying possibilities, a detailed diagnostic workup is required for patient presenting with a working diagnosis of MINOCA (Fig 1).
心臟磁共振成像在非阻塞性冠狀動脈肌鉦蛋白 陽性胸痛中的應用：文獻綜述

李俊賢、蔣碧茜、吳濱濱、㱀隹基、鄭月旺、黃志遠

對於出現急性胸痛和心肌肌鉟蛋白水平升高但血管造影顯示非阻塞性 冠狀動脈的患者的調查和後續管理沒有普遍共識。心藏磁共振成像的 最新技術發展有助了解潛在的病理生理學, 從而為這些患者提供準確 診斷、預後信息和指導。本文回顧支持心藏磁共振成像對急性胸痛和 心肌肌鉟蛋白水平升高但冠狀動脈非阻塞性患者有用的證據, 並提供 對這種成像方式在這種疾病中的作用和未來發展的見解。

\section{Cardiac magnetic resonance imaging protocol}

A targeted CMR imaging protocol tailored to the investigation of MINOCA should require no more than 30 to 40 minutes to perform and is feasible in most patients except the most critically ill. The goal of CMR imaging is to assess cardiac motion and characterise myocardial tissue with full left ventricular coverage, to detect myocardial oedema and necrosis for the diagnoses of various disorders, in particular myocarditis and myocardial infarction. Commonly performed CMR imaging sequences are detailed in the online supplementary Appendix.

Myocardial perfusion assessment with pharmacological stress (eg, adenosine) to evaluate reversible perfusion defects is seldom required in patients with MINOCA except for specific indications such as evaluation of ischaemic extent, and may be contra-indicated in patients with AMI. ${ }^{12}$ Therefore, this evaluation is not recommended as part of the routine assessment.

\section{Timing of cardiac magnetic resonance imaging}

Ideally, CMR imaging should be performed as soon as possible to identify oedema and acute wall motion abnormalities. Although CMR imaging is typically performed after 1 to 4 weeks, the diagnostic value for patients with MINOCA improves significantly when performed within 2 weeks of acute presentation. Studies with longer times to CMR imaging generally show lower sensitivity for demonstrating pathology. One study showed that performing CMR imaging within 2 weeks allowed an underlying cause to be identified in a higher percentage of the study population than if CMR imaging was performed after 2 weeks ( $82 \%$ vs $54 \%$, respectively). ${ }^{13}$ While the Dutch ACS working group recommends CMR imaging within 4 weeks of presentation, ${ }^{11}$ a stricter timeframe of performing CMR imaging within 1 week has been suggested by Ferreira et al. ${ }^{4}$ The 


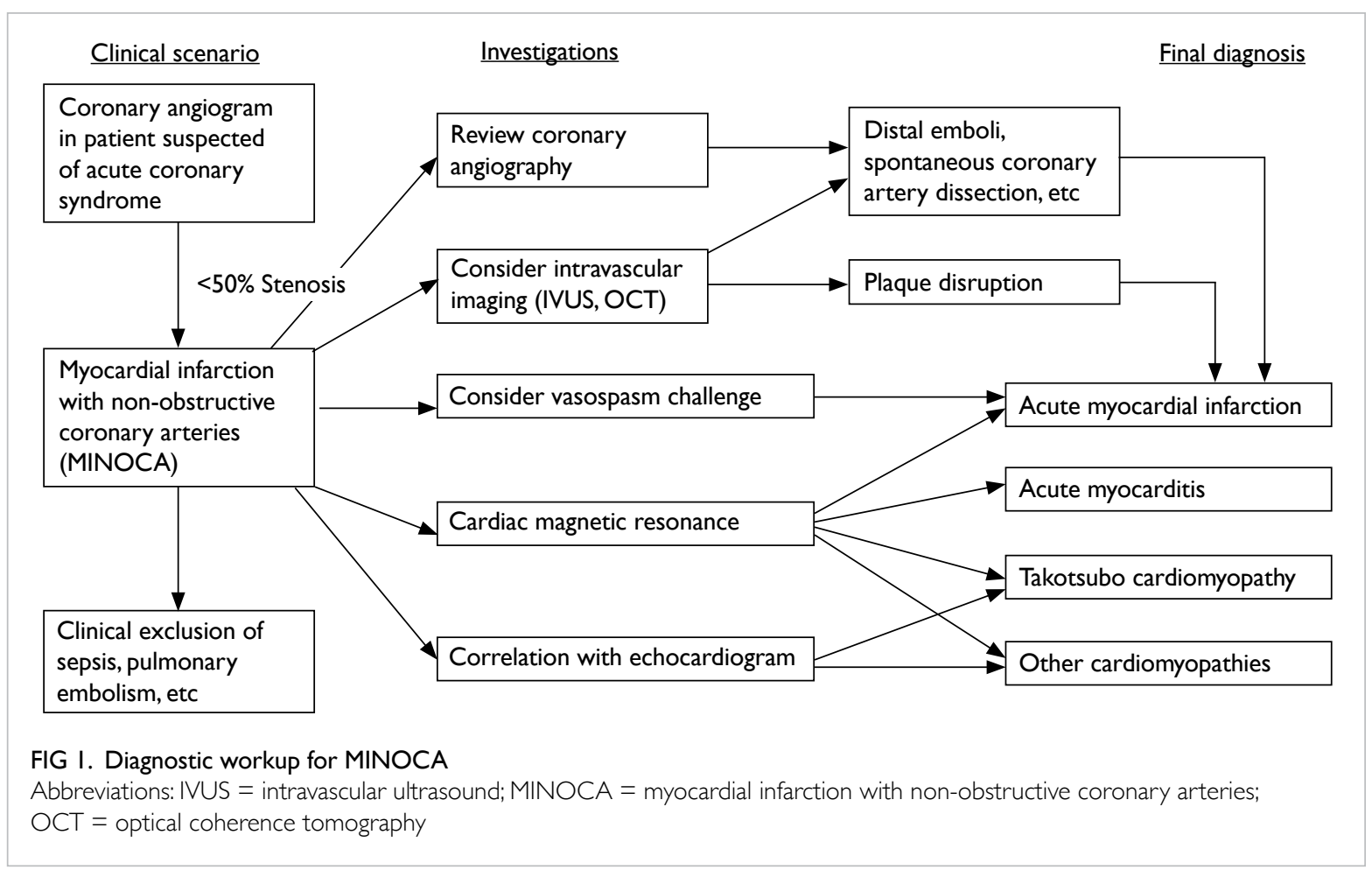

local practice may depend on availability of imaging resources.

\section{Differential diagnoses shown in cardiac magnetic resonance in patients presenting with myocardial infarction with non- obstructive coronary arteries}

In a recent study by Dastidar et al, ${ }^{9}$ the predominant underlying causes of TpNOCA on CMR imaging in 388 consecutive patients were AMI (25\%), myocarditis (25\%), and cardiomyopathy (25\%), although the median time from clinical presentation to CMR imaging was 37 days. In a recent study by Bhatia et $\mathrm{al}^{5}$ involving 215 patients, myocarditis (32\%) was the most common cause, followed by AMI (22\%), cardiomyopathy (20\%) and takotsubo cardiomyopathy (9\%). The strength of the study was the short time interval from clinical presentation to CMR imaging (median: 3.6 days), which could explain the higher proportion of CMR imaging studies resulting in positive diagnosis and the higher incidence of acute myocarditis and takotsubo cardiomyopathy, in which CMR imaging findings may be transient. ${ }^{14}$ Overall, CMR imaging can provide a diagnosis in $30 \%$ to $90 \%$ of patients, as shown in several studies. ${ }^{5,9,15-22}$ The main reasons for the wide range in diagnostic rates among different studies are likely related to the timing of the CMR imaging, heterogeneity of the patient population, different referral patterns and imaging sequences and non-standardised diagnostic criteria. Studies have also shown that CMR imaging results in a change in clinical diagnosis in more than half of patients, ${ }^{20,23}$ and a change in management in $32 \%$ to $42 \%^{23,24}$ of patients.

\section{Takotsubo cardiomyopathy}

Takotsubo cardiomyopathy (Fig 2), also known as stress-induced cardiomyopathy or apical ballooning syndrome, is a reversible cardiomyopathy induced by extreme physical or emotional stress. ${ }^{25-27}$ However, in some patients, no stressful trigger is identified. The exact pathophysiology of takotsubo cardiomyopathy is unclear; however, some postulate that the underlying pathophysiology is related to microvascular vasoreactivity ${ }^{25}$ or hormonal disturbances. ${ }^{26}$ Previously considered a benign condition, an arrhythmogenic risk and increased cardiac mortality are increasingly recognised in patients with takotsubo cardiomyopathy. ${ }^{27}$ In one study, the prevalence of takotsubo cardiomyopathy in patients undergoing CMR imaging was as high as $27 \% .^{20}$ Takotsubo cardiomyopathy is diagnosed according to the proposed Mayo Clinic criteria. ${ }^{28}$ Because these criteria do not focus on the role of CMR imaging, an update in 2016 by the Heart Failure Association of the European Society of Cardiology endorsed the use of CMR imaging for its excellent depiction of right and left ventricular regional wall motion abnormalities and myocardial oedema. ${ }^{29}$ 


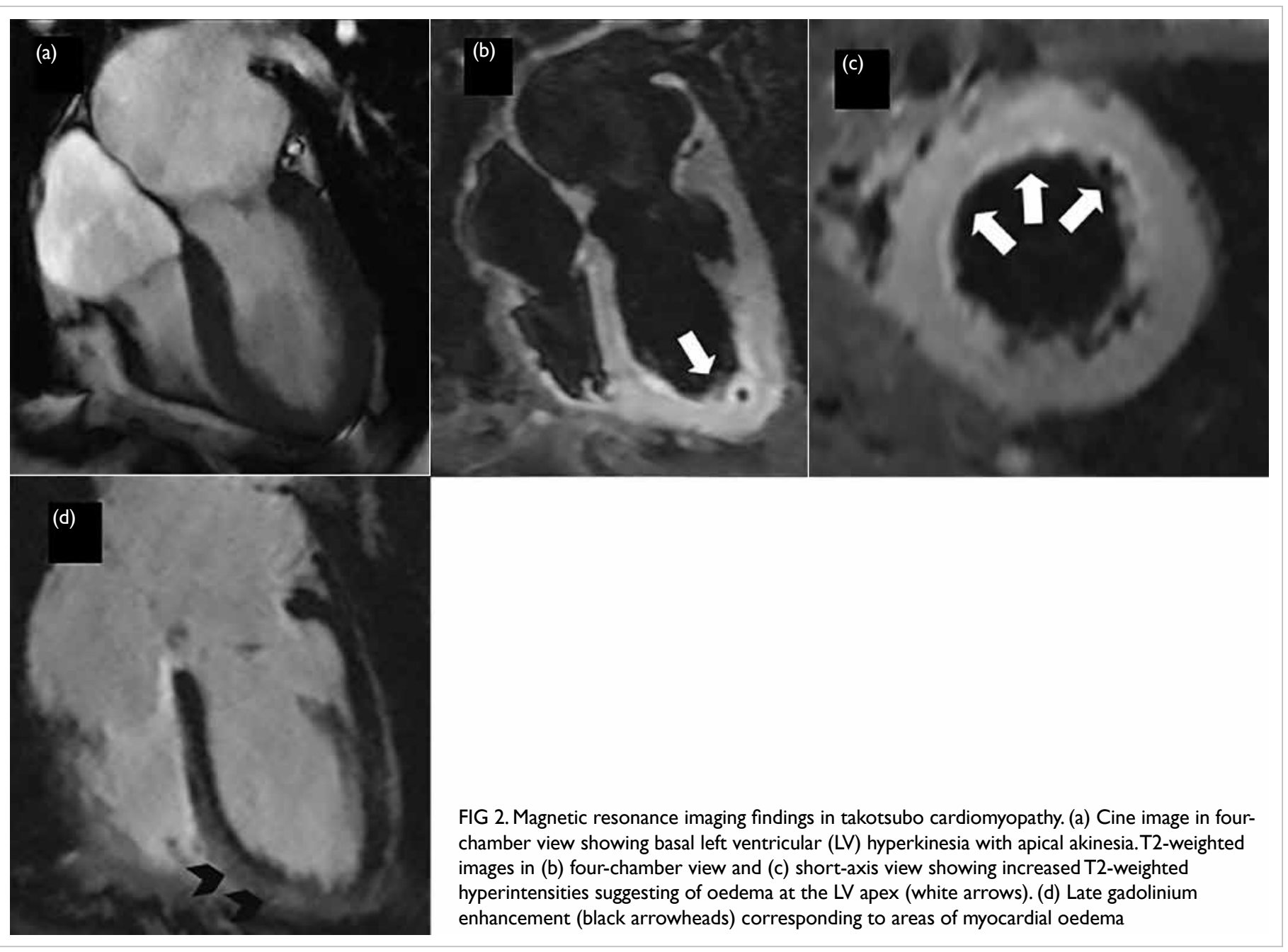

On CMR cine imaging, takotsubo cardiomyopathy has a typical appearance of midcavity to apical akinesia with sparing of basal segments. Although these findings can also be seen in echocardiography and left ventricular angiography, the ability of CMR imaging to assess areas of myocardial oedema and late gadolinium enhancement (LGE), as well as to exclude alternative diagnoses (eg, AMI), makes this an important modality when assessing takotsubo cardiomyopathy. Myocardial oedema (as evidenced using short tau inversion recovery or $\mathrm{T} 2$ mapping techniques) on CMR images correlates with acute myocardial inflammation $^{30}$ and electrographic pattern/ repolarisation indices ${ }^{31}$ in takotsubo cardiomyopathy. The presence of LGE is believed to be transient rather than irreversible. ${ }^{27,32,33}$ Another study showed that LGE in the acute phase was associated with acute cardiogenic shock, higher peak creatine kinase levels, and delayed recovery. ${ }^{34}$ Neil et $\mathrm{al}^{35}$ found that the extent of the increase in T2-weighted signal intensity correlated with myocardial strain and the

release of both catecholamines and $\mathrm{N}$-terminal proB-type natriuretic peptide.

Although no specific treatment is currently available, and spontaneous and complete recovery is often expected, Dastidar et $\mathrm{al}^{9}$ showed that mortality in patients with takotsubo cardiomyopathy can be as high as $15 \%$ over 3 years, rejecting the notion that this is an entirely benign condition. More studies exploring the underlying mechanism and management strategy for takotsubo cardiomyopathy are required.

\section{Acute myocarditis}

Acute myocarditis (Fig 3) accounts for 15\% to $81 \%$ of CMR imaging diagnoses in multiple studies. There are myriad causes of acute myocarditis, including viral infections, autoimmune disease, and toxins. ${ }^{36}$ Patients' clinical courses vary and range from complete recovery to progression to chronic myocarditis and dilated cardiomyopathy. Endomyocardial biopsy remains the gold standard for diagnosing acute myocarditis, although its use 


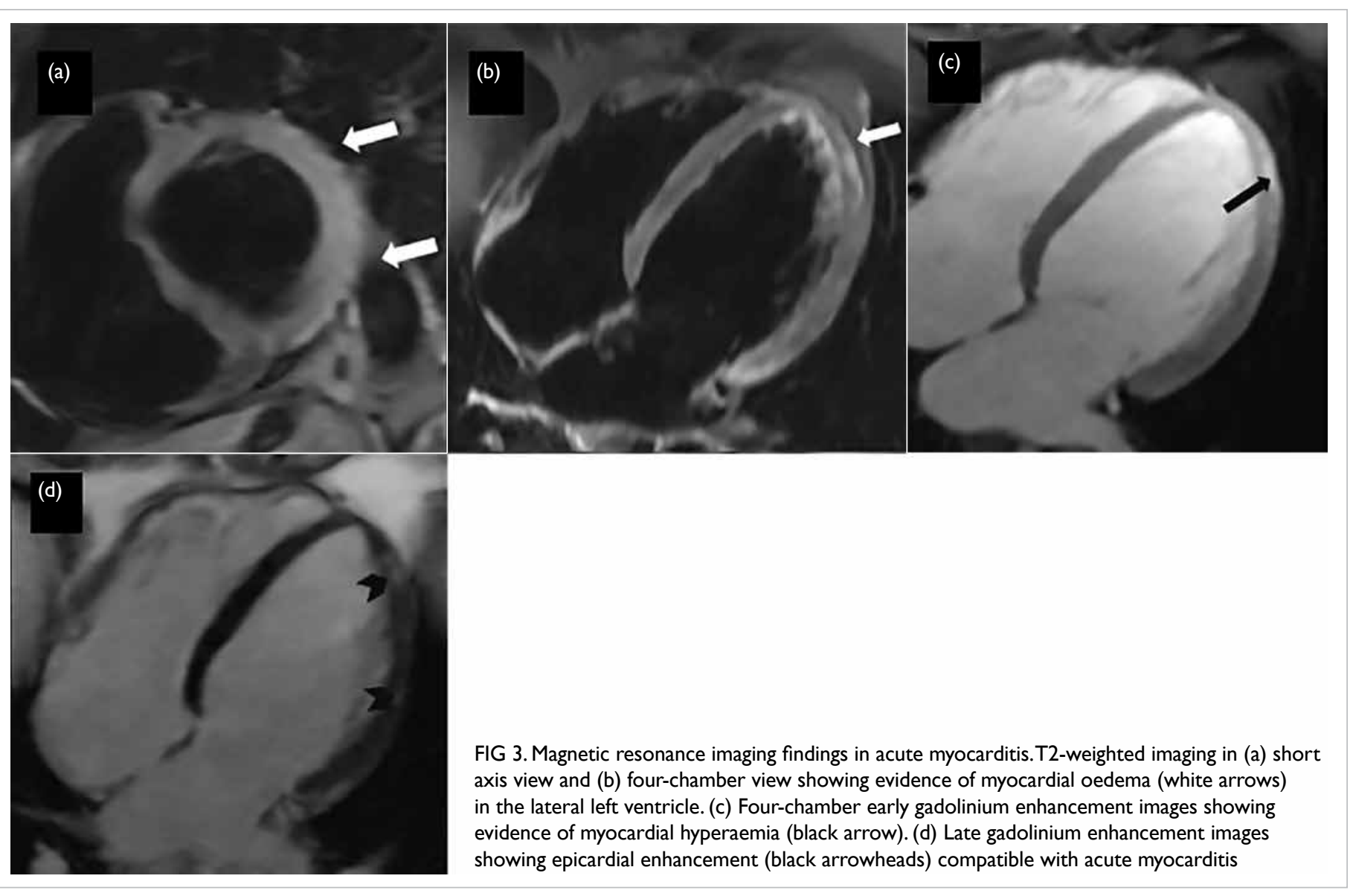

is declining because of its invasiveness and the possibility of sampling error. ${ }^{37}$ A previous study has validated $C M R$ imaging results compared with endomyocardial biopsy ${ }^{38}$ CMR-guided endomyocardial biopsy can improve the diagnostic rate. $^{39,40}$

The CMR diagnosis of acute myocarditis has been made according to the original Lake Louise criteria, which were established in $2009 .{ }^{41}$ These criteria are based on the presence of at least two of three CMR imaging findings: myocardial oedema on T2-weighted images, hyperaemia and capillary leak on EGE, and fibrosis/necrosis on LGE. These criteria have a diagnostic accuracy of $78 \%$ for acute myocarditis. However, co-existing skeletal inflammation may lead to false-negative results in T2 short tau inversion recovery/early gadolinium enhancement images. ${ }^{42}$ With the development of parametric mapping, T1 mapping can establish the diagnosis of myocarditis, even without contrast injection for LGE. ${ }^{43}$ T1 mapping as an individual parameter was found to have superior diagnostic performance for detecting myocarditis compared with T2-weighted oedema imaging. ${ }^{42}$ More recently, a Journal of the American College of Cardiology scientific expert panel updated the use of CMR imaging in myocarditis to include parametric mapping based on at least one T2-based criterion (global or regional increase in myocardial T2 relaxation time or an increased signal intensity in T2weighted CMR images), with at least one T1-based criterion (increased myocardial T1, extracellular volume or LGE). ${ }^{44}$ The inclusion of global or regional $\mathrm{T} 1$ or $\mathrm{T} 2$ myocardial values is expected to improve the diagnostic accuracy of CMR imaging compared with the original Lake Louise criteria. Extracellular volume measurements can also be obtained after contrast administration, adjusting for individual variation in the haematocrit value that may affect the result. The presence of both $\mathrm{T} 2$ - and T1-based criteria is diagnostic of acute myocardial inflammation, while having only one criterion may still support the diagnosis in an appropriate clinical scenario, albeit with less specificity. The updated Lake Louise criteria have been validated by Luetkens et $\mathrm{al}^{45}$ to have better sensitivity than the original Lake Louise criteria ( $88 \%$ vs $73 \%, \mathrm{P}=0.031$ ), with a similar high specificity of $96 \%$.

In addition to diagnosing acute myocarditis, CMR imaging findings have prognostic implications and can help guide patient management. Grun et $\mathrm{al}^{46}$ indicated that LGE was the best independent 
predictor of all-cause mortality and of cardiac mortality in 222 consecutive patients with biopsyproven viral myocarditis. A recent systematic review and meta-analysis by Yang et $\mathrm{al}^{47}$ showed that LGE in patients with myocarditis or suspected myocarditis was significantly associated with MACE (pooled odds ratio $=4.57,95 \%$ confidence interval $[C I]=2.18-9.59 ; \quad P<0.001)$, regardless of the left ventricular ejection fraction. A study by Grani et $\mathrm{al}^{48}$ showed that both the pattern and extent of LGE were significantly associated with MACE. Aquaro et $\mathrm{al}^{49}$ showed that the prognostic value of CMR imaging extends beyond the acute phase, with the presence of LGE with oedema at 6 months being an independent predictor of adverse cardiac events and associated with worse prognosis, especially mid-wall septal patterns in LGE.

More studies are required to determine whether CMR imaging can help differentiate the subtypes of myocarditis (viral, eosinophilic, autoimmune and giant cell myocarditis). ${ }^{44}$

\section{Acute myocardial infarction}

Acute myocardial infarction (Fig 4) was either the most common or second most common aetiology detected by CMR imaging in previous studies, ranging from $11 \%$ to $26 \% .^{5,9}$ The underlying pathophysiological mechanisms included plaque disruption with spontaneous recanalisation, distal embolisation, coronary vasospasm, dissection, or distal small branch disease. On CMR imaging, a classic subendocardial or transmural LGE pattern corresponding to the coronary artery territory, with or without microvascular obstruction, is diagnostic of myocardial infarction. If an infarct is seen, it is essential to review the coronary angiographic images for subtle missed obstructive lesions or coronary artery dissection, and to rule out vasospasm or distal embolisation. Further investigations may depend on clinical suspicion and local practice, and may include intravascular imaging such as optical coherence tomography and intravascular ultrasonography for plaque assessment, provocative tests for coronary vasospasm, echocardiography to identify an embolic source (eg, patent foramen ovale) and thrombophilia screening for hypercoagulable disorders. In Asian populations, vasospastic angina is particularly common and should be carefully managed. ${ }^{50}$ Drugs such as cocaine are well-documented causes of coronary vasospasm and careful elucidation of history is required.

In addition to providing a diagnosis, CMR imaging can also assess myocardial oedema and myocardium at risk in the acute phase to calculate the salvageable area, as well as to assess complications of AMI, such as pseudoaneurysms or intra-cardiac thrombus. Both the presence of a scar and the quantifiable extent of the infarct on LGE have been shown to carry prognostic significance in AMI for predicting morbidity and mortality. ${ }^{51,52}$ The presence of microvascular obstruction is also associated with a worse prognosis. ${ }^{53} \mathrm{~T} 1$ mapping and extracellular volume measurement may be able to differentiate between acute and chronic myocardial infarction. ${ }^{54}$

\section{Non-ischaemic cardiomyopathies}

Hypertrophic cardiomyopathy and dilated cardiomyopathy are the most common forms of non-ischaemic cardiomyopathy presenting with MINOCA (Fig 5). These cardiomyopathies can be diagnosed using CMR imaging according to their morphology and LGE patterns. ${ }^{55}$ The prevalence of non-ischaemic cardiomyopathies in MINOCA varies widely in the literature, and it is unclear whether affected patients were excluded in some studies. Bhatia et $\mathrm{al}^{5}$ showed the highest prevalence of cardiomyopathy among studies that included affected patients, with a prevalence of $20 \%$, making cardiomyopathy the third most common aetiology in MINOCA. A recent study by Dastidar et $\mathrm{al}^{9}$ showed that cardiomyopathy had the worst prognosis among all diagnoses.

A systematic review by Kuruvilla et $\mathrm{a}^{56}$ showed that patients with non-ischaemic cardiomyopathy with LGE had greater all-cause mortality compared with patients without LGE (odds ratio=3.27; 95\% $\mathrm{CI}=1.94-5.51 ; \mathrm{P}<0.00001) .{ }^{56}$ In hypertrophic cardiomyopathy, a meta-analysis ${ }^{57}$ showed that the presence of LGE was associated with an increased risk of sudden cardiac death, heart failure, and cardiovascular mortality and that the extent of LGE was also strongly associated with the risk of sudden cardiac death, suggesting that quantifying LGE is an important tool for risk stratification.

The growing use of parametric mapping will no doubt further enhance the diagnostic capability of CMR imaging in non-ischaemic cardiomyopathies. ${ }^{54}$

\section{Normal/inconclusive cardiac magnetic resonance}

Cardiac magnetic resonance may sometimes not reveal a specific diagnosis, the proportion of which depends on the timing of CMR imaging as well as patients' demographics. Patients with negative CMR imaging findings typically have a lower troponin level. ${ }^{20}$ Occasionally, an infarct may be too small to be visualised by conventional LGE sequences. ${ }^{58}$ Negative CMR imaging findings do not exclude MINOCA. Regardless of whether the underlying cause is identified, the absence of positive CMR imaging findings is associated with a better prognosis. ${ }^{9}$

\section{Managing patients with myocardial infarction with non-obstructive coronary arteries}

Limited guidelines exist regarding the current recommended management of patients with 


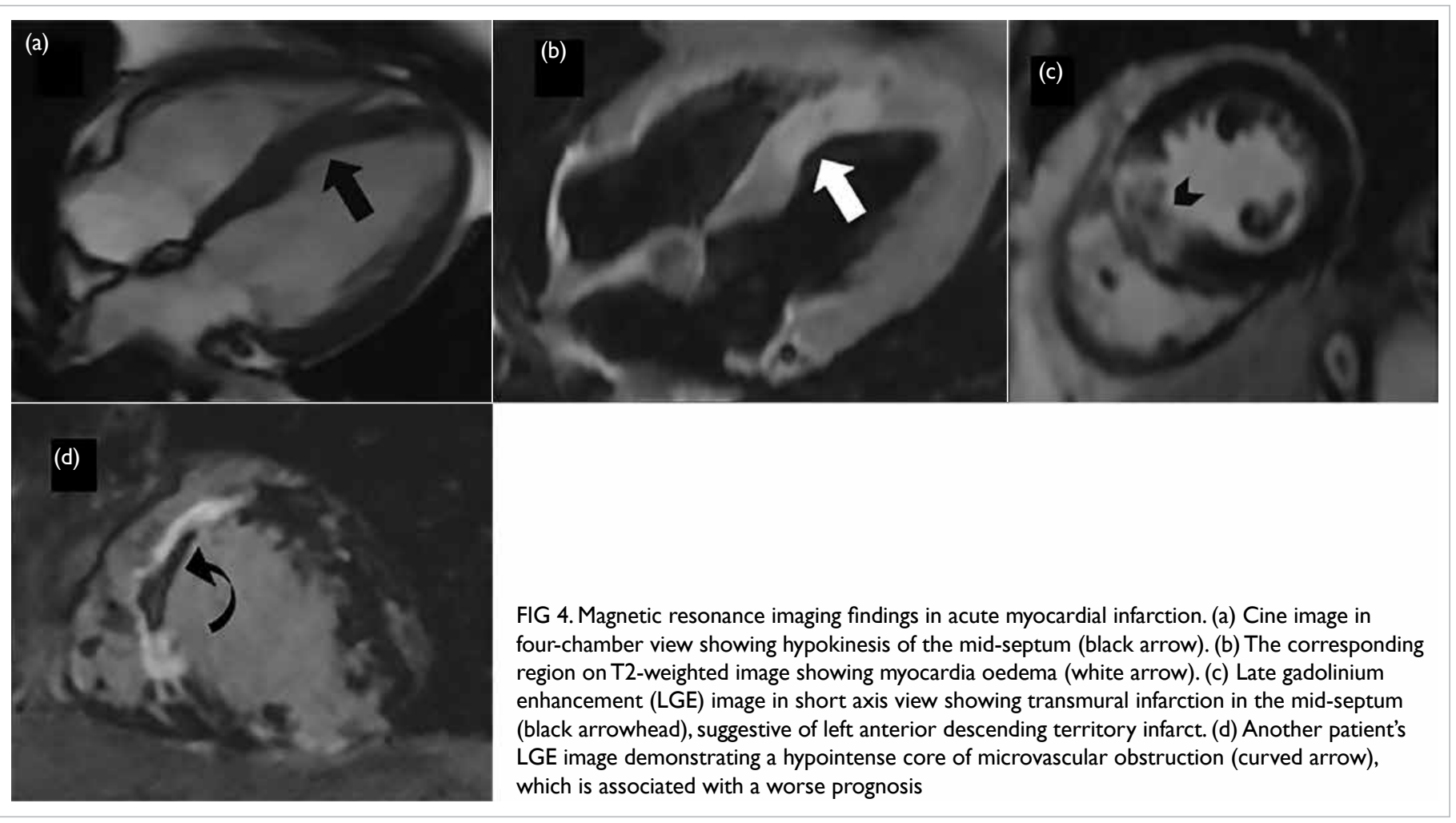

MINOCA, and the management algorithm differs in different centres. Treatment obviously depends on the underlying diagnosis, if identified. In patients without an apparent cause, even by CMR imaging, evidence-based therapies are lacking. Recently, aspirin, statins and calcium channel blockers have been proposed as routine medical treatment in patients with no clear aetiology for elevated troponin on CMR images, to potentially treat underlying thromboembolism, coronary plaque disruption and coronary artery vasospasm. ${ }^{6}$ The evidence for the use of beta-blocker is conflicting. ${ }^{59,60}$ The confirmation of the benefits of these therapies would require a multicentre randomised controlled trial.

\section{Questions to be addressed}

There is a distinct lack of published studies evaluating patients of Asian descent with MINOCA, for whom the local disease spectrum with CMR imaging and the prognostic significance may differ from studies evaluating patients from Western countries, because of differences in the underlying risk factors. It is still unclear in current studies whether performing CMR imaging improves patient outcomes regarding shortening hospital stay, preventing re-admission and lowering MACE and mortality rates. This hypothesis requires validation in further studies in a large patient cohort, with longer follow-up of clinical outcomes. Further studies are also needed to evaluate the relationship between troponin and the extent of LGE, the optimal management pathway and secondary prevention, as well as the role of longterm imaging surveillance to guide management in patients with MINOCA.

\section{Future directions}

With the emergence of novel parametric mapping techniques, namely T1/T2 mapping and extracellular volume measurement, the sensitivity of CMR imaging is expected to improve, as most previous studies did not use T1 and T2 mapping. The optimal mapping techniques and post-processing methods are still being determined, ${ }^{61}$ after which the capability of CMR imaging for diagnosis and prognostication can be further enhanced, providing a better understanding of the underlying pathophysiology in MINOCA. A gadolinium-free or LGE-free protocol combining T2-based CMR imaging with T1 mapping holds significant promise, especially for patients contra-indicated for gadolinium, but further studies are required before this approach can be routinely implemented. Further developments in CMR imaging techniques, such as three-dimensional free-breathing high-resolution LGE, ${ }^{58}$ can lead to a higher rate of definitive myocardial LGE evaluation, thereby reducing the false-negative rate in MINOCA diagnosis. Dedicated rapid CMR imaging protocols or compressed sensing cine can shorten scanning times and permit acquiring diagnostic CMR imaging information even in critically ill patients. 


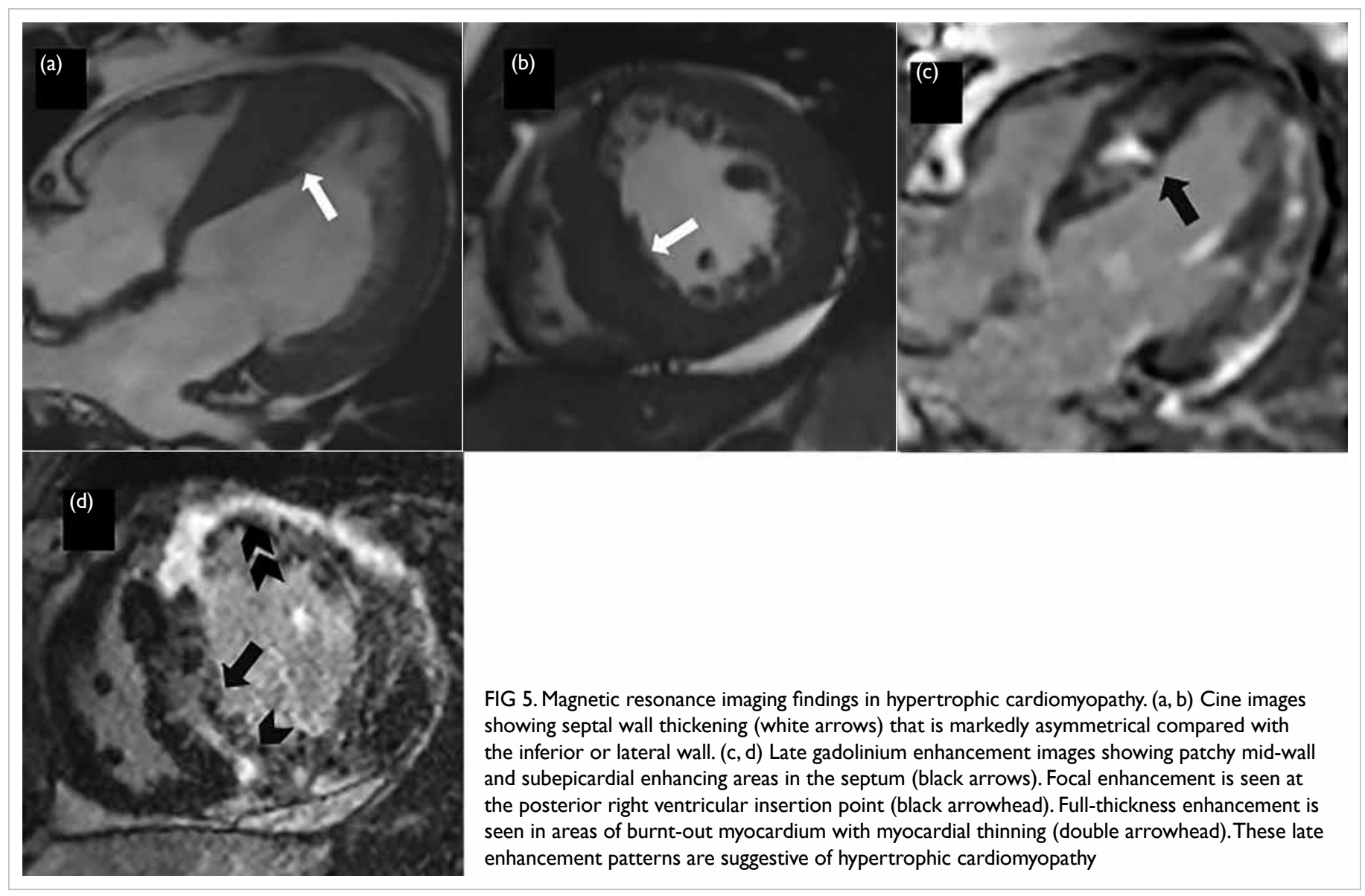

In conclusion, troponin-positive chest pain with non-obstructive coronary arteries should be recognised as a distinct clinical entity that deserves an active search for the underlying cause and a detailed management plan. The absence of obstructive disease on angiography does not necessarily exclude AMI. When performed early in the disease course, CMR imaging is the ideal non-invasive adjunct to conventional cardiac investigations in patients presenting as MINOCA. Cardiac magnetic resonance should be routinely used in these patients for diagnosis and risk stratification to guide further therapy.

\section{Author contributions}

Concept or design: JCY Lee. Acquisition of data: All authors.

Analysis or interpretation of data: JCY Lee, JB Chiang, PP Ng, BCK Chow.

Drafting of the manuscript: JCY Lee, JB Chiang, YW Cheng, CY Wong.

Critical revision of the manuscript for important intellectual content: JCY Lee, YW Cheng, CY Wong.

All authors had full access to the data, contributed to the study, approved the final version for publication, and take responsibility for its accuracy and integrity.

\section{Conflicts of interest}

The authors have disclosed no conflicts of interest.

\section{Funding/support}

This research received no specific grant from any funding agency in the public, commercial, or not-for-profit sectors.

\section{Ethics approval}

The patients were treated in accordance with the Declaration of Helsinki. The patients provided written informed consent for all procedures.

\section{References}

1. Roffi M, Patrono C, Collet JP, et al. 2015 ESC Guidelines for the management of acute coronary syndromes in patients presenting without persistent ST-segment elevation: Task Force for the management of acute coronary syndromes in patients presenting without persistent ST-segment elevation of the European Society of Cardiology (ESC). Eur Heart J 2016;37:267-315.

2. Ibanez B, James S, Agewall S, et al. 2017 ESC Guidelines for the management of acute myocardial infarction in patients presenting with ST-segment elevation: The Task Force for the management of acute myocardial infarction in patients presenting with ST-segment elevation of the European Society of Cardiology (ESC). Eur Heart J 2018;39:119-77.

3. Pasupathy S, Air T, Dreyer RP, Tavella R, Beltrame JF. 
Systematic review of patients presenting with suspected myocardial infarction and nonobstructive coronary arteries. Circulation 2015;131:861-70.

4. Ferreira VM. CMR should be a mandatory test in the contemporary evaluation of 'MINOCA' JACC Cardiovasc Imaging 2019;12:1983-6.

5. Bhatia S, Anstine C, Jaffe AS, et al. Cardiac magnetic resonance in patients with elevated troponin and normal coronary angiography. Heart 2019;105:1231-6.

6. Agewall S, Beltrame JF, Reynolds HR, et al. ESC working group position paper on myocardial infarction with nonobstructive coronary arteries. Eur Heart J 2017;38:143-53.

7. Tamis-Holland JE, Jneid $H$, Reynolds $H R$, et al. Contemporary diagnosis and management of patients with myocardial infarction in the absence of obstructive coronary artery disease: a scientific statement from the American Heart Association. Circulation. 2019;139:e891e908.

8. Thygesen K, Alpert JS, Jaffe AS, et al. Fourth universal definition of myocardial infarction (2018). Circulation 2018;138:e618-e651.

9. Dastidar AG, Baritussio A, De Garate E, et al. Prognostic role of CMR and conventional risk factors in myocardial infarction with nonobstructed coronary arteries. JACC Cardiovasc Imaging 2019;12:1973-82.

10. Pasupathy S, Tavella R, Beltrame JF. Myocardial infarction with nonobstructive coronary arteries (MINOCA): the past, present, and future management. Circulation 2017;135:1490-3.

11. Pustjens TF, Appelman Y, Damman P, et al. Guidelines for the management of myocardial infarction/injury with non-obstructive coronary arteries (MINOCA): a position paper from the Dutch ACS working group. Neth Heart J 2020;28:116-30.

12. Jo Y, Kim J, Park $\mathrm{CH}$, et al. Guideline for cardiovascular magnetic resonance imaging from the Korean Society of Cardiovascular Imaging-part 1: standardized protocol. Korean J Radiol 2019;20:1313-33.

13. Dastidar AG, Singhal P, Rodrigues JC, et al. Improved diagnostic role of CMR in acute coronary syndromes and unobstructed coronary arteries: the importance of timeto-CMR. J Cardiovasc Magn Reson 2015;17(Suppl 1):O87.

14. Sechtem U, Seitz A, Ong P. MINOCA: unravelling the enigma. Heart 2019;105:1219-20.

15. Assomull RG, Lyne JC, Keenan N, et al. The role of cardiovascular magnetic resonance in patients presenting with chest pain, raised troponin, and unobstructed coronary arteries. Eur Heart J 2007;28:1242-9.

16. Monney PA, Sekhri N, Burchell T, et al. Acute myocarditis presenting as acute coronary syndrome: role of early cardiac magnetic resonance in its diagnosis. Heart 2011;97:1312-8.

17. Leurent G, Langella B, Fougerou C, et al. Diagnostic contributions of cardiac magnetic resonance imaging in patients presenting with elevated troponin, acute chest pain syndrome and unobstructed coronary arteries. Arch Cardiovasc Dis 2011;104:161-70.

18. Mahmoudi M, Harden S, Abid N, et al. Troponin-positive chest pain with unobstructed coronary arteries: definitive differential diagnosis using cardiac MRI. Br J Radiol 2012;85:e461-6.

19. Collste O, Sörensson P, Frick M, et al. Myocardial infarction with normal coronary arteries is common and associated with normal findings on cardiovascular magnetic resonance imaging: results from the Stockholm Myocardial Infarction with Normal Coronaries study. J Intern Med 2013;273:189-96.

20. Pathik B, Raman B, Mohd Amin NH, et al. Troponinpositive chest pain with unobstructed coronary arteries: incremental diagnostic value of cardiovascular magnetic resonance imaging. Eur Heart J Cardiovasc Imaging 2016;17:1146-52.

21. Chopard R, Jehl J, Dutheil J, et al. Evolution of acute coronary syndrome with normal coronary arteries and normal cardiac magnetic resonance imaging. Arch Cardiovasc Dis 2011;104:509-17.

22. Laraudogoitia Zaldumbide E, Pérez-David E, Larena JA, et al. The value of cardiac magnetic resonance in patients with acute coronary syndrome and normal coronary arteries [in Spanish]. Rev Esp Cardiol 2009;62:976-83.

23. Dastidar AG, Rodrigues JC, Johnson TW, et al. Myocardial infarction with nonobstructed coronary arteries: impact of CMR early after presentation. JACC Cardiovasc Imaging 2017;10(10 Pt A):1204-6.

24. Gerbaud E, Harcaut E, Coste P, et al. Cardiac magnetic resonance imaging for the diagnosis of patients presenting with chest pain, raised troponin, and unobstructed coronary arteries. Int J Cardiovasc Imaging 2012;28:78394.

25. Galiuto L, De Caterina AR, Porfidia A, et al. Reversible coronary microvascular dysfunction: a common pathogenetic mechanism in apical ballooning or TakoTsubo syndrome. Eur Heart J 2010;31:1319-27.

26. Pizzino G, Bitto A, Crea P, et al. Takotsubo syndrome and estrogen receptor genes: partners in crime? J Cardiovasc Med (Hagerstown) 2017;18:268-76.

27. Dastidar AG, Frontera A, Palazzuoli A, Bucciarelli-Ducci C. Takotsubo cardiomyopathy: unravelling the malignant consequences of a benign disease with cardiac magnetic resonance. Heart Fail Rev 2015;20:415-21.

28. Madhavan M, Prasad A. Proposed Mayo Clinic criteria for the diagnosis of Tako-Tsubo cardiomyopathy and longterm prognosis. Herz 2010;35:240-3.

29. Lyon AR, Bossone E, Schneider B, et al. Current state of knowledge on takotsubo syndrome: a position statement from the Taskforce on Takotsubo Syndrome of the Heart Failure Association of the European Society of Cardiology. Eur J Heart Fail 2016;18:8-27.

30. Iacucci I, Carbone I, Cannavale G, et al. Myocardial oedema as the sole marker of acute injury in takotsubo cardiomyopathy: a cardiovascular magnetic resonance (CMR) study. Radiol Med 2013;118:1309-23.

31. Perazzolo Marra M, Zorzi A, Corbetti F, et al. Apicobasal gradient of left ventricular myocardial edema underlies transient T-wave inversion and QT interval prolongation (Wellens' ECG pattern) in Tako-Tsubo cardiomyopathy. Heart Rhythm 2013;10:70-7.

32. Prasad A, Lerman A, Rihal CS. Apical ballooning syndrome (Tako-Tsubo or stress cardiomyopathy): a mimic of acute myocardial infarction. Am Heart J 2008;155:408-17.

33. Rolf A, Nef HM, Möllmann H, et al. Immunohistological basis of the late gadolinium enhancement phenomenon in tako-tsubo cardiomyopathy. Eur Heart J 2009;30:1635-42.

34. Naruse Y, Sato A, Kasahara K, et al. The clinical impact of late gadolinium enhancement in takotsubo cardiomyopathy: serial analysis of cardiovascular magnetic resonance images. J Cardiovasc Magn Reson 2011;13:67. 
35. Neil C, Nguyen TH, Kucia A, et al. Slowly resolving global myocardial inflammation/oedema in Tako-Tsubo cardiomyopathy: evidence from T2-weighted cardiac MRI. Heart 2012;98:1278-84.

36. Kindermann I, Barth C, Mahfoud F, et al. Update on myocarditis. J Am Coll Cardiol 2012;59:779-92.

37. Leone O, Veinot JP, Angelini A, et al. 2011 Consensus statement on endomyocardial biopsy from the Association for European Cardiovascular Pathology and the Society for Cardiovascular Pathology. Cardiovasc Pathol 2012;21:24574.

38. Lurz P, Eitel I, Adam J, et al. Diagnostic performance of CMR imaging compared with EMB in patients with suspected myocarditis. JACC Cardiovasc Imaging 2012;5:513-24.

39. Mahrholdt H, Goedecke C, Wagner A, et al. Cardiovascular magnetic resonance assessment of human myocarditis: a comparison to histology and molecular pathology. Circulation 2004;109:1250-8.

40. Baccouche H, Mahrholdt H, Meinhardt G, et al. Diagnostic synergy of non-invasive cardiovascular magnetic resonance and invasive endomyocardial biopsy in troponin-positive patients without coronary artery disease. Eur Heart J 2009;30:2869-79.

41. Friedrich MG, Sechtem U, Schulz-Menger J, et al. Cardiovascular magnetic resonance in myocarditis: A JACC White Paper. J Am Coll Cardiol 2009;53:1475-87.

42. Ferreira VM, Piechnik SK, Dall'Armellina E, et al. T1 mapping for the diagnosis of acute myocarditis using CMR: comparison to T2-weighted and late gadolinium enhanced imaging. JACC Cardiovasc Imaging 2013;6:1048-58.

43. Ferreira VM, Piechnik SK, Dall'Armellina E, et al. Native T1-mapping detects the location, extent and patterns of acute myocarditis without the need for gadolinium contrast agents. J Cardiovasc Magn Reson 2014;16:36.

44. Ferreira VM, Schulz-Menger J, Holmvang G, et al. Cardiovascular magnetic resonance in nonischemic myocardial inflammation: expert recommendations. J Am Coll Cardiol 2018;72:3158-76.

45. Luetkens JA, Faron A, Isaak A, et al. Comparison of original and 2018 Lake Louise criteria for diagnosis of acute myocarditis: results of a validation cohort. Radiol Cardiothorac Imaging 2019;1:e190010.

46. Grün S, Schumm J, Greulich S, et al. Long-term follow-up of biopsy-proven viral myocarditis: predictors of mortality and incomplete recovery. J Am Coll Cardiol 2012;59:160415.

47. Yang F, Wang J, Li W, et al. The prognostic value of late gadolinium enhancement in myocarditis and clinically suspected myocarditis: systematic review and metaanalysis. Eur Radiol 2020;30:2616-26.

48. Gräni C, Eichhorn C, Bière L, et al. Prognostic value of cardiac magnetic resonance tissue characterization in risk stratifying patients with suspected myocarditis. J Am Coll Cardiol 2017;70:1964-76.

49. Aquaro GD, Ghebru Habtemicael Y, Camastra G, et al. Prognostic value of repeating cardiac magnetic resonance in patients with acute myocarditis. J Am Coll Cardiol 2019;74:2439-48.

50. Beltrame JF, Crea F, Kaski JC, et al. The who, what, why, when, how and where of vasospastic angina. Circ J 2016;80:289-98.

51. Larose E, Rodés-Cabau J, Pibarot P, et al. Predicting late myocardial recovery and outcomes in the early hours of ST-segment elevation myocardial infarction traditional measures compared with microvascular obstruction, salvaged myocardium, and necrosis characteristics by cardiovascular magnetic resonance. J Am Coll Cardiol 2010;55:2459-69.

52. Yokota H, Heidary S, Katikireddy CK, et al. Quantitative characterization of myocardial infarction by cardiovascular magnetic resonance predicts future cardiovascular events in patients with ischemic cardiomyopathy. J Cardiovasc Magn Reson 2008;10:17.

53. Taylor AJ, Al-Saadi N, Abdel-Aty H, Schulz-Menger J, Messroghli DR, Friedrich MG. Detection of acutely impaired microvascular reperfusion after infarct angioplasty with magnetic resonance imaging. Circulation 2004;109:2080-5.

54. Haaf P, Garg P, Messroghli DR, Broadbent DA, Greenwood JP, Plein S. Cardiac T1 mapping and extracellular volume (ECV) in clinical practice: a comprehensive review. J Cardiovasc Magn Reson 2016;18:89.

55. Karamitsos TD, Francis JM, Myerson S, Selvanayagam JB, Neubauer S. The role of cardiovascular magnetic resonance imaging in heart failure. J Am Coll Cardiol 2009;54:140724.

56. Kuruvilla S, Adenaw N, Katwal AB, Lipinski MJ, Kramer CM, Salerno M. Late gadolinium enhancement on cardiac magnetic resonance predicts adverse cardiovascular outcomes in nonischemic cardiomyopathy: a systematic review and meta-analysis. Circ Cardiovasc Imaging 2014;7:250-8.

57. Weng Z, Yao J, Chan $\mathrm{RH}$, et al. Prognostic value of LGE-CMR in HCM: a meta-analysis. JACC Cardiovasc Imaging 2016;9:1392-402.

58. Lintingre PF, Nivet $H$, Clément-Guinaudeau $S$, et al. High-resolution late gadolinium enhancement magnetic resonance for the diagnosis of myocardial infarction with nonobstructed coronary arteries. JACC Cardiovasc Imaging 2020;13:1135-48.

59. Lindahl B, Baron T, Erlinge D, et al. Medical therapy for secondary prevention and long-term outcome in patients with myocardial infarction with nonobstructive coronary artery disease. Circulation 2017;135:1481-9.

60. Pelliccia F, Pasceri V, Niccoli G, et al. Predictors of mortality in myocardial infarction and nonobstructed coronary arteries: a systematic review and meta-regression. Am J Med 2020;133:73-83.e4.

61. Peker E, Gülpınar B, Elhan AH, Erden MI. Diagnostic accuracy of mapping techniques and postprocessing methods for acute myocarditis. AJR Am J Roentgenol 2020;215:105-15. 